\title{
Recommendations for research priorities in breast cancer by the coalition of cancer cooperative groups scientific leadership council: imaging and local therapy
}

\author{
Joseph A. Sparano $\cdot$ Etta D. Pisano $\cdot$ Julia R. White $\cdot$ Kelly K. Hunt • \\ Eleftherios P. Mamounas • Edith A. Perez • Gabriel N. Hortobagyi • \\ Julie R. Gralow • Robert L. Comis
}

Received: 13 November 2009/Accepted: 14 November 2009/Published online: 19 December 2009

(C) Springer Science+Business Media, LLC. 2009

\begin{abstract}
Imaging and local therapy are important modalities for detection and management of localized breast cancer. Improvements in screening and local therapy have contributed to reduced breast cancer-associated morbidity and mortality. The Coalition of Cancer Cooperative Groups (CCCG) convened the Scientific Leadership Council (SLC) in breast cancer, an expert panel, to identify
\end{abstract}

This study was performed on behalf of the Breast Cancer Scientific Leadership Council of the Coalition of Cancer Cooperative Groups.

\section{J. A. Sparano $(\square)$}

Montefiore Medical Center - Weiler Division, Professor of Medicine and Women's Health, Albert Einstein College of Medicine, 1825 Eastchester Road, 10461 Bronx, NY, USA e-mail: jsparano@montefiore.org

\section{E. D. Pisano}

University of North Carolina, Chapel Hill, NC, USA

\section{J. R. White}

Medical College of Wisconsin Froedtert Hospital, Milwaukee, WI, USA

K. K. Hunt - G. N. Hortobagyi

University of Texas, MD Anderson Cancer Center, Houston, TX, USA

E. P. Mamounas

Aultman Hospital Cancer Center, OH University, Canton, USA

E. A. Perez

Mayo Clinic Jacksonville, Jacksonville, FL, USA

J. R. Gralow

University of Washington School of Medicine, Seattle Cancer

Care Alliance, Seattle, WA, USA

R. L. Comis

Coalition of Cancer Cooperative Groups, Philadelphia, PA, USA priorities for future research and current trials with greatest practice-changing potential. Panelists formed a consensus on research priorities for breast imaging and locoregional therapy, and also identified six trials judged to be of high priority. Current high priority trials included trials determining: (1) the role of accelerated partial breast versus whole-breast radiation (B39), (2) the feasibility, safety, and local and systemic control of small localized breast cancers treated with tumor ablation (Z1072), (3) the role of removal of the primary cancer in selected patients with metastatic disease (E2108), and (4) the clinical and biological effects of pre-operative anti-HER2-directed and ER-directed therapies in localized or locally advanced breast cancer (B41, Z1031, Z1041). Ongoing and future trials will further refine optimal locoregional management, and additional research is required to develop improved screening methods and identify high risk populations most likely to benefit from targeted screening.

Keywords Breast cancer - Imaging - Radiation therapy Local therapy

\section{Introduction}

Imaging and local therapies clearly play important roles in the early detection and management of localized breast cancer. About $60 \%$ of patients present with localized disease, $35 \%$ with regional disease, and 5\% with distant metastases at diagnosis [1]. Breast cancer mortality decreased by about $2.3 \%$ annually in the United States between 1990 and 2000 despite a persistent increase in incidence during this time [2]. This decrease has been attributed to both screening and adjuvant systemic therapy [3]. Subsequently, a decline in age-adjusted incidence rates 
was noted for the first time ever in the United States (U.S.) in 2003 in women aged 50 years or older (and particularly for estrogen receptor (ER)-positive disease) that was attributed to the declining use of hormone replacement therapy (HRT) in this age group [4]. This decline in HRT use followed release of the Women's Health Initiative trial results which indicated a $26 \%$ increase in breast cancer risk and an unfavorable risk-benefit ratio associated with combined estrogen plus progestin in healthy post-menopausal women [5]. Nevertheless, breast cancer remains the most common cause of cancer and second leading cause of cancer death in women in the US [6]. Incremental improvement in detection and management of localized disease achieved through carefully designed clinical trials offers the potential to further reduce breast cancer morbidity and mortality.

The Breast Cancer Scientific Leadership Council (SLC) of the Coalition of Cancer Cooperative Groups (CCCG) is an expert panel that was convened by the CCCG leadership. The objectives of the breast cancer SLC were to evaluate the current state of the art of breast cancer detection, prevention, and management, review the current portfolio of breast cancer clinical trials, identify gaps in the current portfolio, and provide recommendations for prioritizing current and future research. The initial report by the SLC focused on the use of systemic therapy for prevention and treatment of breast cancer [7]. This report focuses on breast cancer detection and imaging, and management of localized disease with surgery and radiation by the same expert panel using methods that were previously described in the prior report.

\section{Imaging}

Current imaging modalities commonly used in practice include mammography, sonography, and magnetic resonance imaging (MRI). The role of each of these modalities in screening is summarized herein and in Table 1.

\section{Screening mammography}

Screening mammography (using conventional analog film) reduces breast cancer mortality by $26 \%$ in women aged $50-74$ [8], and by $18 \%$ in women aged 40-50 [9]. The observed decline in breast cancer mortality in the U.S. which became evident in the 1990s has been attributed in part to the widespread use of screening mammography [3]. However, mammography is only about $80-90 \%$ sensitive in patients with clinically detectable disease, and its lack of specificity leads to approximately $2-5$ patients requiring biopsy for benign conditions for every cancer diagnosed $[10,11]$. In addition, declining mammography screening rates between 1999 and 2002 that were first reported in 2007 have raised concern that this may eventually lead to increased breast cancer mortality [12]. Factors contributing to these declining mammography screening rates include lack of insurance coverage, a poor understanding of the value of screening, and fear of cancer diagnosis. An Institute of Medicine report in 2004 expressed concern about shortage of breast imaging specialists, and recommended that researchers and technology developers focus their efforts on developing tools to identify those women who would derive the most benefit from screening [13].

Table 1 Role of imaging in breasts cancer screening

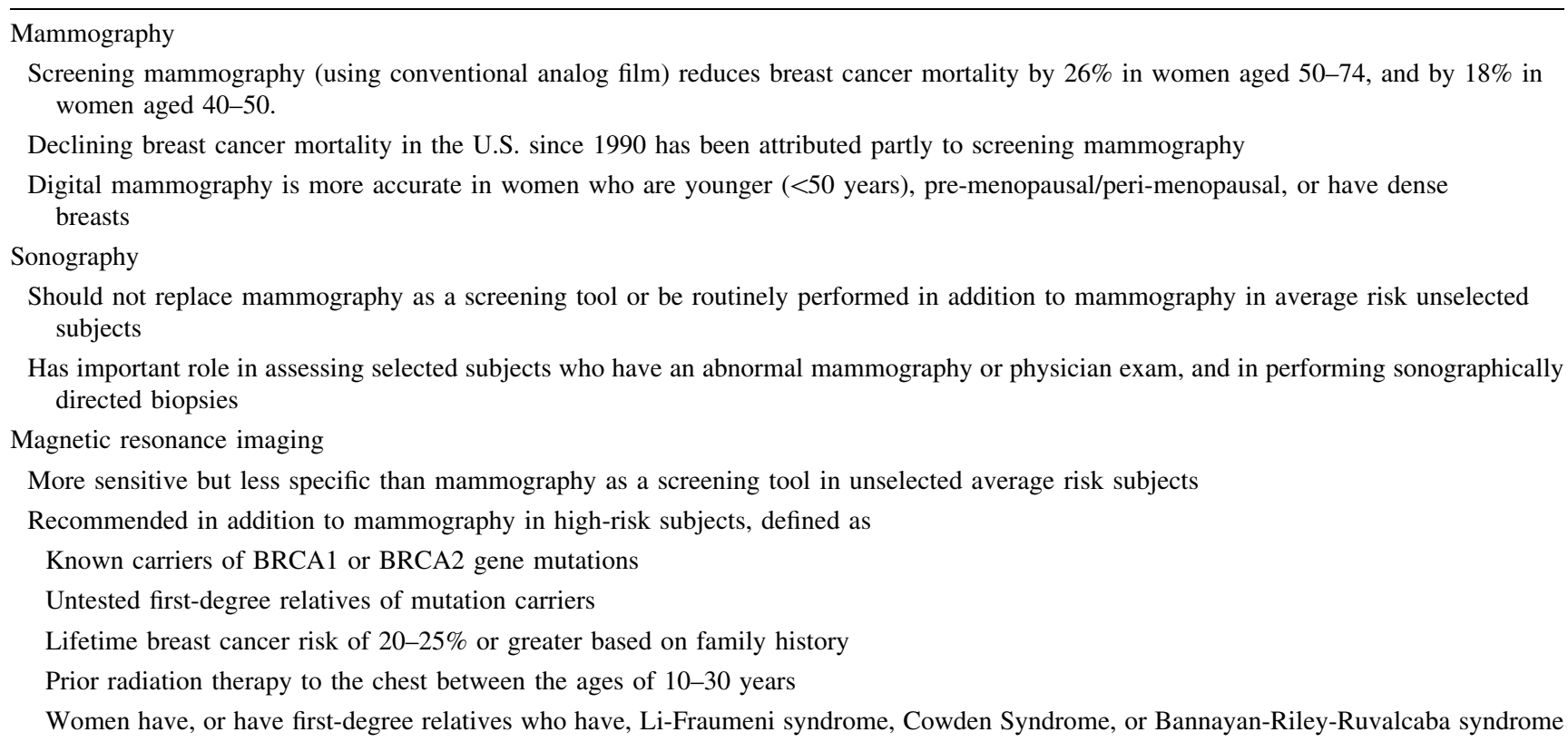


Digital mammography

Digital mammography separates the processes of image acquisition, image display, and storage, allowing each to be optimized [14]. It allows the brightness and contrast of the displayed image to be manipulated independently from the process of acquiring the image, and, in general, uses less radiation than film-screen mammography [15]. The Digital Mammographic Imaging Screening Trial (DMIST) prospectively enrolled 49,528 women in the United States and Canada who underwent both film-screen mammography and digital mammography. Each woman's mammograms were interpreted independently by two different radiologists [16]. Although there was no significant difference in diagnostic accuracy between the two modalities in the overall trial population, digital mammography exhibited better accuracy in women younger than age 50 , in premenopausal/peri-menopausal women, and in those with extremely dense or heterogeneously dense breasts. The difference in accuracy in these subgroups was due to improved sensitivity of digital mammography without a difference in specificity. An ongoing cost analysis from DMIST suggests that digital mammography is more expensive than film mammography, but despite its 1.5-4fold higher acquisition costs, there was greater cost-effectiveness in women younger than age 50 [17]. Digital mammography also allows new applications that may improve early diagnosis, such as contrast-enhanced digital mammography and "telemammography"-the electronic transmission of a digital image from a local physician's office or community hospital to a radiologist specializing in breast imaging, without loss of image quality. Digital mammography, however, requires rigorous training for the radiologist and has a learning curve. The addition of intravenous contrast during digital mammography may help increase the detection rate of occult lesions in mammographically dense breasts [18].

\section{Sonography}

Screening ultrasound has been evaluated in single-center studies and retrospective reviews, which have indicated a $0.3 \%$ cancer detection rate in unselected healthy subjects or healthy subjects with dense breast tissue [19-21]. A small prospective study compared the cancer yield of MRI, mammography, and ultrasound $(N=171)$ [22]. Ultrasound produced a diagnostic yield of $0.6 \%$ compared with $3.5 \%$ for MRI and $1.2 \%$ for mammography. Ultrasound was also less sensitive than MRI and mammography at detecting lesions in a group of women with BRCA1 or BRCA2 mutations [23]. The American College of Radiology Imaging Network (ACRIN) 6666 trial evaluated ultrasound and mammography at baseline, 12 months, and 24 months, and was designed to compare sonography versus mammography versus both used together [24]. Preliminary results in 2,504 women indicated that ultrasound can detect mammographically occult early stage cancer, but with a substantial risk of false positives $[25,26]$. Cancer yield was 4.2 per 1000 for ultrasound alone, 8.0 per 1000 for mammography alone, and 12.4 per 1000 for ultrasound plus mammography, which results in a supplemental yield of 4.4 per 1000 women (95\% CI: $1.2-7.6$ per 1000) screened when added to mammography. The diagnostic accuracy of ultrasound plus mammography was $93 \%$ compared with $79 \%$ for mammography alone $(P=.004)$. Of the 168 women $(6.7 \%)$ who actually underwent biopsy based on ultrasound results, $7.1 \%$ had proven cancer, $3.6 \%$ had atypical lesions, and $89 \%$ had benign results. Moreover, initial results found that the addition of ultrasound screening to mammography resulted in additional biopsies in $6.7 \%$ of women, of whom only $7.1 \%$ actually had cancer. Therefore, the evidence indicates that sonography should not replace mammography as a screening tool, and should not be routinely performed in all patients in addition to mammography. However, it does play an important role in assessing selected patients who have an abnormal mammographic exam or physical exam, and in performing sonographically directed biopsies.

\section{Magnetic resonance imaging}

The use of magnetic fields instead of X-rays produces detailed cross-sectional images, and sensitivity is enhanced by the use of gadolinium for contrast enhancement. For high-risk women, MRI is more sensitive than mammography for in situ and invasive disease (71.1 vs. $40.0 \%)$, and also when only invasive disease is considered (79.5 vs. $33.3 \%$ ) [27]. In addition, invasive cancers detected by MRI are smaller and more often associated with negative axillary nodes [28-30]. MRI is also more sensitive for detecting in situ disease than mammography (90 vs. 58\%), with MRI being more sensitive for high grade disease and mammography for low grade disease [31]. However, although MRI does appear to be more sensitive for both invasive and in situ disease, mammography is more specific (95.0 vs. 89.8\%) [27]. Thus, the issue becomes weighing the greater sensitivity of MRI against the greater specificity of mammography. Based on a recent systematic review of five accuracy studies, the addition of MRI to conventional screening tests facilitates early cancer detection in young, high-risk women [32]. Another role for MRI screening includes screening of the contralateral breast in women with a breast cancer diagnosis, in which a contralateral cancer was detected in $3.1 \%$ of patients which was not otherwise detected by physical exam or mammography [33]. MRI is clearly superior to mammography and 
indicated in women with BRCA1 or BRCA2 mutations; in this setting, MRI detected $77 \%$ of cancers compared with $36 \%$ detected by mammography, $33 \%$ by ultrasound, and $9 \%$ by clinical examination [23].

The American Cancer Society (ACS) recommends annual MRI screening as an adjunct to mammography for known carriers of BRCA1 or BRCA2 gene mutations, untested first-degree relatives of mutation carriers, and women with lifetime breast cancer risk of 20-25\% or greater based on family history models (summarized in Table 1) [34]. Other indications include prior radiation therapy to the chest between ages 10 and 30 years, and women who have first-degree relatives with Li-Fraumeni syndrome, Cowden syndrome, or Bannayan-Riley-Ruvalcaba syndrome. Because the data are insufficient to recommend for or against annual MRI for women with a lifetime risk between 15 and $20 \%$, the ACS recommends that physicians should consider annual MRI screening added to annual mammography on an individual basis for these women. In women with a personal history of breast cancer, there are insufficient data to make firm guidelines regarding the use of MRI in screening. Annual MRI screening is not recommended for women with a lifetime risk $<15 \%$.

Recent studies have indicated increasing rates of mastectomy (including contralateral prophylactic mastectomy) $[35,36]$, and some evidence indicates that this may be attributed to the increased use of MRI [37]. In addition, others have shown that MRI does not contribute to improved outcomes in those who have breast conservation [38], and may result in inappropriate preference for mastectomy in patients who would otherwise have been suitable candidates for breast conservation [39]. Therefore, studies that go beyond increased sensitivity and demonstrate clinical benefit (improved local control rate or survival) are needed.

\section{Investigational screening modalities}

Investigational screening tools include methods for anatomic, functional, and combined imaging. Positron emission tomography (PET) imaging after injection of radioactive tracer ([18]F-fluorodeoxyglucose [FDG]) combines functional and anatomic imaging, which may enable improved cancer detection in dense breasts, improved differentiation of cancers from scar tissue, and improved definition of extent of disease prior to surgery. In a prospective, multicenter trial, PET imaging had 93\% sensitivity and $83 \%$ specificity for index lesions (i.e., the lesions that prompted study entry) [40]. Larger studies to assess the accuracy, costeffectiveness, and receiver-operator characteristic (ROC) performance measurements associated with FDG-PET imaging for screening are needed. Tomosynthesis is a technique to improve visibility of lesions by reducing image complexity due to overlap of tissue structures. It uses a series of images from different angles that are recorded on a single digital detector, with computer processing used to clarify the image. At this time, data on dose and reproducibility are minimal and not yet published in the peer-reviewed literature [41]. ACRIN is considering investigating tomosynthesis with intravenous contrast versus MRI (Pisano ED: personal communication).

Important clinical research questions for screening

At present, digital mammography is poised to replace conventional film-screen mammography because of its advantages in image capture, manipulation, storage, and transmission. In some patient subsets, it is very accurate in detecting breast cancer. Adjunctive screening with MRI also has become established as having value for high-risk women. Nonetheless, important research questions remain which are shown in Table 2. Questions include which modality is best suited for individual patients? Which is best for women with dense breasts, or for younger women? Which is best for those with a prior breast cancer diagnosis? Who may safely be screened less often or starting at an older age? Who should be screened more often or before age 50? Can a combination of serum or genetic tests be used to help identify women for more aggressive screening?

Table 2 Research recommendations in breast cancer screening

Development of screening tools that will identify women who would derive the most benefit. Researchers and technology developers should focus their efforts on developing tools to identify women who would derive the most benefit from BC screening. How can we identify which screening tool will be best for individual patients? Which tool is best for women with dense breasts, for younger women, or for those with established BC? Which women could be safely screened less often or starting at an older age? Which women could be screened more often or before age 50 ?

Cost-effectiveness of new screening technologies. The cost of all new technologies must be addressed, that is, how cost-effective are these new tools in terms of cancer identified and lives saved? Do incremental benefits of adjunctive screening translate to a cost-effective advantage across the population of women? If not, for which populations will there be a cost-effective advantage?

Risk of radiation exposure. Most of the existing and investigational screening modalities involve exposure to radiation. Further research is needed to determine the relative risk of radiation exposure with new technologies. Moreover, can new tools be developed to reduce women's radiation exposure and risk of false positives? 
Two other key issues include cost and radiation exposure. How cost-effective are these new strategies? Do the incremental benefits of adjunctive screening translate into a cost-effective advantage across the population of women, and, if not, for which populations will they do so? With regard to radiation exposure, what is the relative risk of radiation exposure with new technologies? It has been established that radiation exposure with digital mammography, in general, is lower than that of film-screen mammography [42]. But, for example, how much increased risk is imparted for high-risk women screened with digital mammography? Can new tools be developed to reduce women's radiation exposure and risk of false positives? Do the benefits of additional layers of screening with contrastenhanced digital mammography or PET imaging outweigh the risks of additional radiation exposure? Only long-term studies can answer these questions.

\section{Surgery}

Surgery has the dual goal of removing the tumor from the breast and regional lymph nodes and providing accurate staging [42]. Surgery may also have a role in selected patients with metasatic disease [43]. Lumpectomy is a less disfiguring procedure, but requires accurate assessment of the extent of the disease in order to ensure complete tumor removal with an acceptable cosmetic result. The presence of tumor-free margins has been associated with lower local recurrence rates after breast conserving surgery [43], but has not been defined consistently. Absence of tumor cells at the inked resection margin has been defined as acceptable in studies performed by the National Surgical Adjuvant Breast Project (NSABP) [44], but more widely negative margins may contribute to lower local recurrence rates, especially for women with locally advanced disease treated with preoperative chemotherapy. Questions remain about how best to measure the actual tumor extent, as well as what are the optimal margins of normal tissue around the tumor that should be removed. New methods to assess tumor margins status are of interest $[45,46]$.

\section{Tumor ablation}

Ablation of the primary tumor using radiofrequency, lasergenerated heat, or cryosurgery is a recent surgical innovation that, while locally effective, is still considered experimental [47]. The presumed benefits of tumor ablation are its minimally invasive nature and potential to destroy tumors without the need for resecting normal surrounding breast tissue, potentially producing minimal cosmetic sequelae. Although results to date indicate that tumor ablation may have the potential to replace surgical resection for the treatment of small primary breast tumors, further studies are needed to determine whether the use of tumor ablation alone will result in similar long-term outcomes. Limitations include imprecise identification of the location and extent of the primary tumor, application to multifocal disease, the potential for higher local failure rates, the loss of anatomic information regarding tumor size, and biomarkers, and post-procedure surveillance that may be impaired by fat necrosis and procedure-associated calcification. Before ablative therapies can be adopted widely, carefully conducted trials evaluating local, regional, and systemic control are needed.

\section{Surgery after neoadjuvant therapy}

Neoadjuvant chemotherapy is currently the preferred approach for locally advanced disease, and is a reasonable option for those with large tumors who could benefit from tumor cytoreduction prior to surgery in order to facilitate breast conservation. Numerous studies have shown comparable benefit from systemic chemotherapy in patients with operable disease, whether given post- or pre-operatively [48]. Some studies have shown higher local recurrence rates with breast conservation after neoadjuvant chemotherapy, which may be in part related to difficulty in localizing the tumor by examination or imaging, or tumors that decrease in size in a honey-comb pattern rather than a concentric pattern. Some have advocated placement of clips in the tumor bed prior to (or during) neoadjuvant therapy, which has resulted in improved local control rates in some retrospective studies [49].

\section{Sentinel lymph node biopsy}

Multiple clinical trials have demonstrated the feasibility and efficacy of lymphatic mapping and sentinel lymph node (SLN) biopsy in patients with operable breast cancer [50-56]. On average, the rate of false negative SLN biopsy is $8-10 \%$. Sentinel lymph node biopsy has become widely adopted for patients with operable breast cancer and a clinically negative axilla. In the largest randomized trial to date, NSABP B-32, over 5600 women with clinically negative axilla were randomly assigned to receive SLN biopsy with axillary lymph node dissection (ALND) or SLN biopsy alone [53]. When they become available, longterm results from that trial will compare the two approaches relative to disease-free survival, overall survival, and axillary recurrence rates [57].

Several techniques for intraoperative SLN evaluation have been investigated to guide the decision to excise additional lymph nodes and to aid in patient staging. These techniques include imprint cytology (touch preparation), frozen sectioning, rapid intraoperative cytokeratin staining 
in conjunction with imprint cytology, and frozen sectioning, scrape preparation, or a combination of these techniques. Currently, the widely practiced intraoperative methods are imprint cytology and frozen sectioning. However, both of these methods have limited sensitivity with wide performance variability [58-60]. Recently, the FDA approved the first molecular-based diagnostic test for detecting whether breast cancer has metastasized to lymph nodes. The GeneSearch ${ }^{\mathrm{TM}}$ Breast Lymph Node (BLN) Assay (Veridex LLC, Raritan, NJ) was found to have greater sensitivity than traditional intraoperative methods for detecting the spread of breast cancer to the lymph nodes. Results from a prospective multisite study that enrolled 416 patients revealed that the BLN Assay detected approximately $10 \%$ more metastases than frozen sectioning and nearly $30 \%$ more than touch preparation [61]. Overall, the assay accurately detected $98 \%$ of metastases $>2 \mathrm{~mm}$ and $88 \%$ of metastases $\geq 0.2 \mathrm{~mm}$ [61]. These data support the use of the BLN Assay as an intraoperative molecular test that can reduce the need for second surgeries to complete axillary dissection in SLN-positive patients.

Sentinel lymph node biopsy for patients receiving neoadjuvant therapy

There is no consensus on the use and optimal timing of SLN biopsy in patients who are candidates for neoadjuvant chemotherapy, which is known to downstage the disease in axillary lymph nodes in up to $30-40 \%$ of patients [62]. Results from NSABP B-27 suggest that SLN biopsy after neoadjuvant therapy is accurate ( $85 \%$ identification rate, $11 \%$ false negative) [62], and a meta-analysis of available studies suggests that SLN biopsy after chemotherapy is a reliable indicator of the axillary lymph node status following neoadjuvant therapy [63]. As it currently stands, there remains considerable debate whether performing SLN biopsy before or after neoadjuvant chemotherapy will result in differences in the accuracy of the procedure, the staging information obtained, and whether some patients can be spared ALND based on the findings of SLN biopsy. It also remains unclear whether the decision to use postoperative irradiation should be based on the baseline lymph node status or status after neoadjuvant chemotherapy.

Role of surgery in metastatic disease

Another outstanding surgical question is whether and which women who present with metastatic disease benefit from primary tumor resection. Although some retrospective analyses suggest a survival advantage for patients who undergo primary breast tumor removal, the results are subject to selection biases [62]. Thus, clinical trials are needed to better define the role of surgery in this setting. In particular, will reducing primary tumor burden prolong survival in any particular subpopulation of patients? Conversely, is there benefit to surgical removal of oligometastases before or after systemic therapy for metastatic breast cancer?

Summary of surgical therapy advances and important clinical research questions

Future research priorities and current high priority trial involving surgery identified by the SLC are summarized in Table 3. Some of these trials are driven by evaluating the effects of neoadjuvant systemic therapy on eradication of microscopic disease from the breast using HER2-directed therapy and endocrine therapy HER2-positive disease (B41, Z1041) and ER-positive disease (Z1031), respectively. Others evaluate the role and timing of surgery in metastatic disease (E2108), or the role of cryoablation in patients with small tumors less than $2 \mathrm{~cm}$ (Z1072). Current surgical trends continue to evolve toward less aggressive and less disfiguring procedures. Research into radiologic and biologic markers may provide more precise measures of tumor extent and may help identify patients whose tumors are more likely to respond to particular neoadjuvant (or adjuvant) systemic therapies.

\section{Radiation}

The goal of radiation therapy is to eradicate microscopic cancer cells that remain in the breast, chest wall or regional nodes following surgery \pm chemotherapy. The optimal reduction in local regional recurrence from radiation therapy following breast conserving surgery or mastectomy can result in a decrease in long-term breast cancer mortality as demonstrated in meta-analyses [64]. Radiation therapy to the breast is standard for most women who undergo lumpectomy and for many women with node-positive disease who undergo mastectomy. Following breast conserving surgery, the treated area includes the breast, and regional lymph nodes in selected patients with node-positive breast cancer. Post-mastectomy radiation is directed to the chest wall and regional nodal areas and is generally recommended for women with four or more positive lymph nodes, and 1-3 positive lymph nodes with high-risk features (e.g., large primary tumors, high nodal ratio, etc.). The relative advantages of radiation have been offset by an increased risk of cardiac death noted in long-term breast cancer survivors treated with older radiation techniques, but is no longer apparent with modern techniques for delivering radiation $[65,66]$. Recent advances have focused on radiating less breast and regional tissue (i.e., partial breast irradiation) by either external beam or local 
Table 3 Research recommendations and high priority studies in surgery for early and advanced disease

Extent of primary tumor. It is imperative to understand the extent of the primary breast tumor to ensure that complete tumor removal is achieved. We should determine the best way to measure the actual tumor extent and the optimal margins of normal tissue around the tumor.

Optimal timing of SLN biopsy. There is considerable debate regarding whether to perform SLN biopsy before or after neoadjuvant chemotherapy. Further research is needed to determine whether there would be differences in the accuracy of the procedure, staging information obtained, and outcome. Moreover, we should determine whether some patients can be spared ALND based on the SLN biopsy findings.

Optimal second-line neoadjuvant or adjuvant chemotherapy in patients from neoadjuvant trials who do not respond to first-line therapy. Further research is needed to evaluate novel chemotherapy regimens either alone or in combination with innovative targeted agents to determine the optimal adjuvant treatment in patients who did not respond to first-line treatment.

Role of surgery in metastatic disease. Retrospective analyses suggest a survival advantage for patients with metastatic disease who undergo primary tumor resection. There are also indications that removal/sterilization of oligometastases combined with systemic therapy might result in long-term disease control. We need prospective trials to determine whether reducing tumor burden prolongs survival.

Identifying subpopulations of women who need more or less aggressive therapy. Several neoadjuvant trials prospectively stratified by biological or radiographic markers are ongoing. These types of trial will be important in determining meaningful clinical endpoints and biomarkers and how biomarkers should be used in directing treatment decisions. Correlation of the impact of these markers in the neoadjuvant versus outcome in the adjuvant setting is needed.

\begin{tabular}{|c|c|c|c|}
\hline Study & Target accrual & Study population & Research question \\
\hline NSABP-B-41 (NCT00486668) & 522 & HER2-positive & $\begin{array}{l}\text { Efficacy of doxorubicin/cyclophosphamide followed by } \\
\text { weekly paclitaxel plus trastuzumab, lapatinib or the } \\
\text { combination }\end{array}$ \\
\hline ACOSOG-Z1031 (NCT00265759) & 375 & $\begin{array}{l}\text { ER-positive/node- } \\
\text { positive or negative }\end{array}$ & Efficacy of exemestane vs. letrozole vs. anastrozole \\
\hline ACOSOG-Z1041 (NCT00513292) & 270 & HER2-positive & $\begin{array}{l}\text { Efficacy of 5-fluorouracil, epirubicin, and cyclophosphamide } \\
\text { Given before (without trastuzumab) or after } \\
\text { (with trastuzumab) } \\
\text { Neoadjuvant treatment with paclitaxel-trastuzumab }\end{array}$ \\
\hline ACOSOG-Z1072 (NCT00723294) & 99 & $T<2 \mathrm{~cm}$ disease & Phase II study of cryoablation \\
\hline E2108 & 840 & $\begin{array}{l}\text { Metastatic and } \\
\text { local disease }\end{array}$ & $\begin{array}{l}\text { Randomized trial evaluating the role of early vs. delayed } \\
\text { local therapy }\end{array}$ \\
\hline
\end{tabular}

delivery (e.g., brachytherapy, Mammosite), and on improving techniques for more precise delivery of radiation, such as CT volume-based treatment planning, intensity-modulated radiation therapy (IMRT), 3D conformal radiation therapy (3D-CRT), and respiratory gating.

\section{Accelerated partial breast irradiation}

Accelerated partial breast irradiation (APBI), which is currently being intensely investigated, uses brachytherapy or 3D-CRT to treat the $1-2 \mathrm{~cm}$ of breast tissue surrounding the surgical cavity instead of the entire breast. Among the key clinical questions addressed in these trials is the determination of the optimal volume of breast to irradiate after lumpectomy in order to maintain local control rates equivalent to standard whole-breast irradiation (WBI). The presumption of clinical benefit with APBI is based on the finding that most true in-breast recurrences are found near the lumpectomy cavity and that in-breast recurrences in other breast quadrants are most likely new primary tumors whose incidence may not be affected by post-lumpectomy irradiation. Which breast cancer patients can achieve optimal local control from APBI remains unknown pending the completion of CTs. Most patients that have received APBI are considered "low risk." The American Society of Breast Surgeons' criteria for APBI include age older than 50 , tumor size less than $2 \mathrm{~cm}$, invasive or in situ disease, 2-mm tumor-free margins, and node-negative disease [67]. The American Society of Radiation Oncology (ASTRO) consensus statement on APBI defines this group as older than age 60 , tumor size $<2 \mathrm{~cm}$, negative margins, invasive ductal cancer, and node negative [67].

Accelerated partial breast irradiation is an attractive alternative to traditional WBI because it requires a shorter course of therapy (5-8 days), potentially allows additional breast-sparing surgery if a recurrence occurs, and allows irradiation to be completed prior to administration of adjuvant chemotherapy for women who require both modalities (in addition to improved patient convenience and presumably lower costs). APBI may be delivered using four different established techniques, including multicatheter brachytherapy systems, external beam 3D-CRT, the MammoSite brachytherapy balloon, and intraoperative radiation therapy. Several CTs using APBI are underway, and early results are available for some. Results from RTOG 95-17 show that low- and high-dose-rate ABPI with 
interstitial brachytherapy have acceptable acute and late adverse effects, similar to WBI [68, 69]. RTOG 0319 demonstrated that 3D-CRT is feasible and provides reproducible results across institutions. In a study using 3D-CRT to deliver APBI for women with early stage breast cancer treated with breast-conserving therapy $(N=91 ; 46$ evaluated for $\geq 2$ years), no local recurrences were reported after a median follow-up of 24 months, with 12 women followed for 4 years or more [70]. Ninety-one percent of women who were evaluated rated their cosmetic results as good or excellent. The MammoSite Radiation Therapy System received FDA approval in 2002 to deliver brachytherapy to breast cancer patients after lumpectomy. This system was found to be well tolerated and easier to place than interstitial brachytherapy in a phase 2 study of 32 women, but radiation overdoses to the skin and late skin damage also were noted in some cases [68]. Five-year results of another study that treated 43 women using MammoSite balloon brachytherapy to deliver APBI demonstrated excellent local control among women with earlystage invasive ductal breast cancer [71]. Patients received $34 \mathrm{~Gy}$ in 10 fractions over 5 days. Minimal toxicities were reported, and $83.3 \%$ of women rated the cosmetic outcome as good or excellent

The use of APBI, with its attractive qualities of shorter treatment duration and exposure of less normal tissue to radiation, may help to raise interest-and thus improve accrual-in CTs designed to answer questions about radiation therapy.

\section{External beam whole-breast irradiation}

After breast-conservation surgery, standard 2-dimensional (2D) WBI has a well-established track record of good local control, good cosmetic results, and low toxicity [67]. The advent of newer radiation modalities and methods raises questions as to whether 2D WBI delivered over 5-7 weeks is still the standard of care for women with breast cancer, given that modalities such as 3D-CRT and IMRT demonstrate improved targeting to avoid harm to normal tissue and methods such as hypo-fractionation can reduce the burden of care by decreasing the overall treatment time by $30 \%$.

One approach could be to implement newer radiation therapy techniques to improve the therapeutic ratio of WBI, such as the use of image guidance for tumor bed localization and IMRT to improve dose homogeneity, and hypo-fractionation to accelerate treatment [72]. Use of IMRT reduces both acute and late toxicity rates in initial clinical trials [73-75]. Still, its application varies widely due to limited data of optimal target volumes, normal tissue constraints, radiation therapy parameters, and local control. Hypo-fractionated WBI delivers WBI in 16 treatments as opposed to 25-28 and has demonstrated good local control and toxicity [76, 77]. However, these studies used older WBI methods, excluded patients based on size, and did not routinely use a supplemental radiation dose (boost) to the highest-risk tissue around the surgical cavity proven in CT to reduce in-breast recurrence in many patients [75].

By using IMRT, an accelerated WBI treatment can be delivered in a shorter, 3-week course that also allows delivery of a higher biologic dose to the surgical cavity [78]. A recent phase $1 / 2$ study reported that delivering accelerated WBI with an integrated higher dose to the surgical cavity using IMRT to patients in the prone position is feasible and greatly reduces the amount of radiation exposure to the heart and lungs [79]. These help to reduce the burden of care, potentially improving acceptability, and access. The shorter course of therapy and reduced need for post-treatment care may offset the higher costs of IMRT.

Radiation in locally advanced breast cancer

Use of APBI and IMRT is associated with less toxicity to normal tissue [75], with good cosmetic results [70, 71]. This leaves open the clinical question of whether the use of these techniques can reduce the known toxicity and sequelae of radiation therapy for locally advanced breast cancer. A study in three patients with locally advanced breast cancer found that use of IMRT (using segmented multileaf collimation) required a lower dose of radiation and produced less exposure of the heart and lung tissue to radiation than a traditional wide-tangential approach [80]. Before recommendations can be made about the use of 3DCRT and/or IMRT for post-mastectomy radiation therapy or regional nodal irradiation in stage IIB-III disease, clinical trials will need to define optimal methods and demonstrate acceptable, local regional control, and toxicity rates.

Summary of radiation therapy advances and important clinical research questions

Research priorities in radiation therapy identified by the SLC are summarized in Table 4. The advent and adoption of new techniques for delivering radiation therapy promise shorter courses of therapy and less toxicity to skin and normal heart and lung tissue. However, outstanding questions remain and must be addressed in randomized CTs. In the next $1-2$ years, a priority is to complete NSABP B-39/ RTOG 0413 (clinicaltrials.gov; NCT00103181), a phase 3 randomized study of adjuvant WBI versus adjuvant APBI in stage $0-$ II breast cancer; this trial was amended after too many low-risk patients were recruited, requiring exclution of additional low risk subjects and increasing the sample size in women with DCIS or stage I/II breast cancer. 
Table 4 Research recommendations and high priority studies in radiation therapy

Optimal breast volume to irradiate after lumpectomy. The RTOG 0413/NSABP B-39 trial is under way to examine the best way to define optimal breast volume to irradiate after lumpectomy.

Incorporate translational studies. There is a need to identify biological and genetic markers for tumor response and patient toxicity to improve individualization of $\mathrm{BC}$ treatment. How can we best identify patients who benefit from radiation therapy prior, or after, surgery?

Evolving radiation technologies and modalities. How do we implement novel radiation technologies and modalities? Should they be implemented? Does utilization of these modalities improve patient outcomes?

\begin{tabular}{llcc}
\hline Study & Target accrual & Study population & Research question \\
\hline $\begin{array}{l}\text { NSABP B-39/RTOG 0413 } \\
\text { (NCT00103181) }\end{array}$ & 4300 & $\begin{array}{c}\text { DCIS or Stage I/II } \\
\text { breast cancer }\end{array}$ & $\begin{array}{c}\text { Conventional whole-breast } \\
\text { irradiation vs. accelerated partial } \\
\text { breast irradiation }\end{array}$
\end{tabular}

The primary endpoint is in-breast recurrence; also assessed will be overall, recurrence-free, and distant disease-free survival. Accelerated partial breast irradiation can be administered by interstitial catheter-based brachytherapy, MammoSite balloon-based brachytherapy, or external beam 3D-CRT.

There are many unanswered questions regarding radiation use in stage IIB-III locally advanced breast cancer for which CTs are under development. These questions address optimizing and individualizing local therapy for breast cancer patients: Is it feasible to implement IMRT/3D-CRT conformal therapy for regional nodal irradiation in locally advanced breast cancer and reduce toxicity? Can radiation following mastectomy or nodal irradiation following breast conservation be safely omitted based on complete response to neoadjuvant chemotherapy in stage II-IIIA breast cancer? Will the development of preoperative radiation methods following neoadjuvant chemotherapy for locally advanced breast cancer incrementally improve complete response rates, allow for more breast conservation, facilitate immediate breast reconstruction; and can biomarkers for radiation response be identified? In addition, ablative image-guided radiation (SBRT) for metastases has been developed. Clinical trials designed to evaluate whether the use of this technology can change the outcome for oligometastatic disease are warranted. A major priority for future radiation-oncology protocols is to incorporate translational studies to identify biological and genetic markers for tumor response and patient toxicity to better individualize breast cancer treatment.

Acknowledgments We thank Donna Marinucci, Robert Catalano, PharmD, Diane Colaizzi, MA, Karen Creamer, RN Allan Wolkin, $\mathrm{RPh}$, and Alyson Fick for providing subject matter expertise. We also thank Edisa Gozun, PharmD, and Roseanne Degnan, PharmD, from Scientific Connexions for their research and assistance. Partial support for the SLC in Breast Cancer provided by Amgen, Bristol Myers-Squibb, Genentech, GlaxoSmithKline, Novartis, Pfizer, and sanofi-aventis.

\section{Appendix: Scientific leadership council members}

Scientific leadership council in breast cancer

Information included in this manuscript was taken from the consensus-based document finalized September, 2008Research priorities in breast cancer: recommendations of the scientific leadership council in breast cancer of the coalition of cancer cooperative groups.

Julie Gralow, MD, Co-Chair

University of Washington, School of Medicine

Seattle Cancer Care Alliance, Seattle, WA

Edith Perez, MD, Co-Chair

Mayo Clinic

Jacksonville, FL

Donald Berry, PhD

University of Texas, MD Anderson Cancer Center

Houston, TX

Lisa Carey, $\mathrm{MD}$

University of North Carolina

Lineberger Comprehensive Cancer Center

Chapel Hill, NC

David Cella, PhD

Center on Outcome Research-Education

Evanston Northwestern Healthcare

Evanston, IL

Marc Citron, MD

Albert Einstein College of Medicine

Lake Success, NY

Nancy Davidson, MD

University of Pittsburgh Cancer Institute

Pittsburgh, PA

Gabriel Hortobagyi, MD F.A.C.P.

University of Texas, MD Anderson Cancer Center

Houston, TX

Clifford Hudis, MD

Memorial Sloan Kettering Cancer Center

New York, NY 
Kelly Hunt, MD

University of Texas, MD Anderson Cancer Center

Houston, TX

Eleftherios Mamounas, MD

Aultman Hospital Cancer Center

Canton, $\mathrm{OH}$

Kathy Miller, MD

Indiana University Cancer Center

Indianapolis, IN

Etta Pisano, MD

University of North Carolina

Chapel Hill, NC

Kathleen Pritchard, MD

Toronto-Sunnybrook Regional Cancer Center

Toronto, Ontario

George Sledge, Jr., MD

Indiana University Cancer Center

Indianapolis, IN

Joseph Sparano, MD

Montefiore Medical Center-Weiler Division

Bronx, NY

Julia White, MD

Medical College of Wisconsin, Froedtert Hospital

Milwaukee, WI

Eric Winer, MD

Dana-Farber Cancer Institute

Boston, MA

Antonio Wolff, MD

Johns Hopkins Kimmel Cancer Center

Baltimore, MD

William Wood, MD

Emory University Hospital

Atlanta, GA

Robin Zon, MD

Michiana Hematology-Oncology PC

South Bend, IN

Note added in proof The U.S. Prevent Services Task Force recently published an update of its screening recommendations for breast cancer which have generated considerable controversy. Major changes in the Task Force recommendations compared with their prior recommendations in 2002 included (1) a recommendation against routine screening mammography in women aged 40-49 years (rather than a recommendation to begin at 40 in the 2002 recommendations), (2) a recommendation for biennial screening in women age 50-74 years (rather than annual in the 2002 recommendations). The revised recommendations were based in part upon new information obtained from two studies it commissioned, including a targeted systemic evidence review of 6 selected questions relating to the benefits and harms of screening, and a decision analysis that used population modeling techniques to compare the expected health outcomes and resource requirements of starting and ending mammography screening at different ages and using annual versus biennial screening intervals. The panel also identified research needs and gaps, and called for a series of large randomized clinical trials.
US Preventive Services Task Force. Screening for breast cancer: U.S. Preventive Services Task Force recommendation statement. Ann Intern Med. 2009 Nov 17;151(10):716-26, W-236. PubMed PMID: 19920272.

Nelson HD, Tyne K, Naik A, Bougatsos C, Chan BK, Humphrey L; U.S. Preventive Services Task Force. Screening for breast cancer: an update for the U.S. Preventive Services Task Force. Ann Intern Med. 2009 Nov 17;151(10):727-37, W237-42. PubMed PMID: 19920273

\section{References}

1. American Cancer Society (2007) Breast cancer facts and figures 2007-2008. American Cancer Society, Inc, Atlanta

2. Stewart SL, King JB, Thompson TD et al (2004) Cancer mortality surveillance-United States, 1990-2000. MMWR Surveill Summ 53:1-108

3. Berry DA, Cronin KA, Plevritis SK et al (2005) Effect of screening and adjuvant therapy on mortality from breast cancer. N Engl J Med 353:1784-1792

4. Ravdin PM, Cronin KA, Howlader N et al (2007) The decrease in breast-cancer incidence in 2003 in the United States. N Engl J Med 356:1670-1674

5. Rossouw JE, Anderson GL, Prentice RL et al (2002) Risks and benefits of estrogen plus progestin in healthy postmenopausal women: principal results from the women's health initiative randomized controlled trial. JAMA 288:321-333

6. Jemal A, Siegel R, Ward E et al (2009) Cancer statistics, 2009. CA Cancer J Clin 59:225-249

7. Sparano JA, Hortobagyi GN, Gralow JR, Perez EA, Comis RL (2009) Recommendations for research priorities in breast cancer by the coalition of Cancer Cooperative Groups Scientific Leadership Council: systemic therapy and therapeutic individualization. Breast Cancer Res Treat. doi:10.1007/s10549009-0433-y

8. Kerlikowske K, Grady D, Rubin SM et al (1995) Efficacy of screening mammography. A meta-analysis. JAMA 273:149154

9. Hendrick RE, Smith RA, Rutledge JH III et al (1997) Benefit of screening mammography in women aged 40-49: a new metaanalysis of randomized controlled trials. J Natl Cancer Inst Monogr 22:87-92

10. Kopans DB (2003) Mammographic screening for breast cancer. $\mathrm{N}$ Engl J Med 349:610-612 (author reply 610-612)

11. Kopans DB, Feig SA (1998) False positive rate of screening mammography. N Engl J Med 339:562-564

12. Centers for Disease Control, Prevention (2007) Use of mammograms among women aged $>$ or $=40$ years-United States 2000-2005. MMWR Morb Mortal Wkly Rep 56:49-51

13. Joy JE, Penhoet EE, Petitti DB (eds) (2004) Saving women's lives: strategies for improving breast cancer detection and diagnosis. The National Academies Press, Washington, DC

14. Pisano ED, Yaffe MJ (2005) Digital mammography. Radiology 234:353-362

15. Pisano ED, Acharyya S, Cole EB et al (2009) Cancer cases from ACRIN digital mammographic imaging screening trial: radiologist analysis with use of a logistic regression model. Radiology 252:348-357

16. Pisano ED, Gatsonis C, Hendrick E et al (2005) Diagnostic performance of digital versus film mammography for breastcancer screening. N Engl J Med 353:1773-1783

17. Tosteson AN, Stout NK, Fryback DG et al (2008) Cost-effectiveness of digital mammography breast cancer screening. Ann Intern Med 148:1-10 
18. Jong RA, Yaffe MJ, Skarpathiotakis M et al (2003) Contrastenhanced digital mammography: initial clinical experience. Radiology 228:842-850

19. Buchberger W, DeKoekkoek-Doll P, Springer P et al (1999) Incidental findings on sonography of the breast: clinical significance and diagnostic workup. AJR Am J Roentgenol 173:921927

20. Kaplan SS (2001) Clinical utility of bilateral whole-breast US in the evaluation of women with dense breast tissue. Radiology 221:641-649

21. Kolb TM, Lichy J, Newhouse JH (2002) Comparison of the performance of screening mammography, physical examination, and breast US and evaluation of factors that influence them: an analysis of 27, 825 patient evaluations. Radiology 225:165-175

22. Lehman CD, Isaacs C, Schnall MD et al (2007) Cancer yield of mammography, MR, and US in high-risk women: prospective multi-institution breast cancer screening study. Radiology 244:381-388

23. Warner E, Plewes DB, Hill KA et al (2004) Surveillance of BRCA1 and BRCA2 mutation carriers with magnetic resonance imaging, ultrasound, mammography, and clinical breast examination. JAMA 292:1317-1325

24. Berg WA, Blume JD, Cormack JB, Mendelson EB, Lehrer D, Böhm-Vélez M, Pisano ED, Jong RA, Evans WP, Morton MJ, Mahoney MC, Larsen LH, Barr RG, Farria DM, Marques HS, Boparai K, ACRIN 6666 Investigators (2008) Combined screening with ultrasound and mammography vs mammography alone in women at elevated risk of breast cancer. JAMA 299(18):2151-2163

25. Berg W (2007) Yield of screening breast ultrasound and mammography compared to mammography alone: results of first screen in ACRIN (American College of Radiology Imaging Network, the Radiological Society of North America (RSNA) 93rd Scientific Assembly and Annual Meeting. Chicago, Illinois

26. Berg W (2007) Risk of false positives with supplemental screening breast US: results from the first screen in the ACRIN (American College of Radiology Imaging Network), the Radiological Society of North America (RSNA) 93rd Scientific Assembly and Annual Meeting. Chicago, Illinois

27. Kriege M, Brekelmans CT, Boetes C et al (2004) Efficacy of MRI and mammography for breast-cancer screening in women with a familial or genetic predisposition. N Engl J Med 351:427-437

28. Brekelmans CT, Seynaeve C, Bartels CC et al (2001) Effectiveness of breast cancer surveillance in BRCA1/2 gene mutation carriers and women with high familial risk. J Clin Oncol 19:924930

29. Chart PL, Franssen E (1997) Management of women at increased risk for breast cancer: preliminary results from a new program. CMAJ 157:1235-1242

30. Lalloo F, Boggis CR, Evans DG et al (1998) Screening by mammography, women with a family history of breast cancer. Eur J Cancer 34:937-940

31. Kuhl CK, Schrading S, Wardelmann E et al (2007) Magnetic resonance imaging versus mammography for diagnosing ductal carcinoma in situ. J Clin Oncol 25:1504 (suppl; abstr 1504)

32. Lord SJ, Lei W, Craft P et al (2007) A systematic review of the effectiveness of magnetic resonance imaging (MRI) as an addition to mammography and ultrasound in screening young women at high risk of breast cancer. Eur J Cancer 43:1905-1917

33. Lehman CD, Gatsonis C, Kuhl CK et al (2007) MRI evaluation of the contralateral breast in women with recently diagnosed breast cancer. N Engl J Med 356:1295-1303

34. Saslow D, Boetes C, Burke W et al (2007) American Cancer Society guidelines for breast screening with MRI as an adjunct to mammography. CA Cancer J Clin 57:75-89
35. Tuttle TM, Habermann EB, Grund EH et al (2007) Increasing use of contralateral prophylactic mastectomy for breast cancer patients: a trend toward more aggressive surgical treatment. J Clin Oncol 25:5203-5209

36. Tuttle TM, Jarosek S, Habermann EB et al (2009) Increasing rates of contralateral prophylactic mastectomy among patients with ductal carcinoma in situ. J Clin Oncol 27:1362-1367

37. Katipamula R, Degnim AC, Hoskin T et al (2009) Trends in mastectomy rates at the Mayo Clinic Rochester: effect of surgical year and preoperative magnetic resonance imaging. J Clin Oncol 27:4082-4088

38. Solin LJ, Orel SG, Hwang WT et al (2008) Relationship of breast magnetic resonance imaging to outcome after breast-conservation treatment with radiation for women with early-stage invasive breast carcinoma or ductal carcinoma in situ. J Clin Oncol 26:386-391

39. Bleicher RJ, Ciocca RM, Egleston BL et al (2009) Association of routine pretreatment magnetic resonance imaging with time to surgery, mastectomy rate, and margin status. J Am Coll Surg 209:180-187 (quiz 294-295)

40. Berg WA, Weinberg IN, Narayanan D et al (2006) High-resolution fluorodeoxyglucose positron emission tomography with compression ("positron emission mammography") is highly accurate in depicting primary breast cancer. Breast J 12:309-323

41. Poplack SP, Tosteson TD, Kogel CA et al (2007) Digital breast tomosynthesis: initial experience in 98 women with abnormal digital screening mammography. AJR Am J Roentgenol 189:616-623

42. Vazquez B, Rousseau D, Hurd TC (2007) Surgical management of breast cancer. Semin Oncol 34:234-240

43. Morrow M (2007) Improved survival in metastatic breast cancer following total excision of the primary tumor. Nat Clin Pract Oncol 4(1):14-15

44. Lee MC, Newman LA (2007) Management of patients with locally advanced breast cancer. Surg Clin North Am 87:379-398 (ix)

45. Haka AS, Volynskaya Z, Gardecki JA et al (2006) In vivo margin assessment during partial mastectomy breast surgery using Raman spectroscopy. Cancer Res 66:3317-3322

46. Bickford LR, Agollah G, Drezek R, Yu TK (2009) Silica-gold nanoshells as potential intraoperative molecular probes for HER2-overexpression in ex vivo breast tissue using near-infrared reflectance confocal microscopy. Breast Cancer Res Treat. doi: 10.1007/s10549-009-0408-z

47. Singletary SE, Fornage BD, Sneige N et al (2002) Radiofrequency ablation of early-stage invasive breast tumors: an overview. Cancer J 8:177-180

48. Waljee JF, Newman LA (2007) Neoadjuvant systemic therapy and the surgical management of breast cancer. Surg Clin North Am 87:399-415 (ix)

49. Oh JL, Nguyen G, Whitman GJ et al (2007) Placement of radiopaque clips for tumor localization in patients undergoing neoadjuvant chemotherapy and breast conservation therapy. Cancer 110:2420-2427

50. Albertini JJ, Lyman GH, Cox C et al (1996) Lymphatic mapping and sentinel node biopsy in the patient with breast cancer. JAMA 276:1818-1822

51. Bedrosian I, Reynolds C, Mick R et al (2000) Accuracy of sentinel lymph node biopsy in patients with large primary breast tumors. Cancer 88:2540-2545

52. Giuliano AE, Kirgan DM, Guenther JM et al (1994) Lymphatic mapping and sentinel lymphadenectomy for breast cancer. Ann Surg 220:391-398 (discussion 398-401)

53. Krag DN, Julian TB, Harlow SP et al (2004) NSABP-32: phase III, randomized trial comparing axillary resection with sentinal 
lymph node dissection: a description of the trial. Ann Surg Oncol 11:208S-210S

54. McMasters KM, Tuttle TM, Carlson DJ et al (2000) Sentinel lymph node biopsy for breast cancer: a suitable alternative to routine axillary dissection in multi-institutional practice when optimal technique is used. J Clin Oncol 18:2560-2566

55. O'Hea BJ, Hill AD, El-Shirbiny AM et al (1998) Sentinel lymph node biopsy in breast cancer: initial experience at Memorial Sloan-Kettering Cancer Center. J Am Coll Surg 186:423-427

56. Wong SL, Edwards MJ, Chao C et al (2002) The effect of prior breast biopsy method and concurrent definitive breast procedure on success and accuracy of sentinel lymph node biopsy. Ann Surg Oncol 9:272-277

57. Newman LA, Mamounas EP (2007) Review of breast cancer clinical trials conducted by the National Surgical Adjuvant Breast Project. Surg Clin North Am 87:279-305 (vii)

58. Creager AJ, Geisinger KR, Perrier ND et al (2004) Intraoperative imprint cytologic evaluation of sentinel lymph nodes for lobular carcinoma of the breast. Ann Surg 239:61-66

59. Shiver SA, Creager AJ, Geisinger K et al (2002) Intraoperative analysis of sentinel lymph nodes by imprint cytology for cancer of the breast. Am J Surg 184:424-427

60. Creager AJ, Geisinger KR, Shiver SA et al (2002) Intraoperative evaluation of sentinel lymph nodes for metastatic breast carcinoma by imprint cytology. Mod Pathol 15:1140-1147

61. Blumencranz P, Whitworth PW, Deck K et al (2007) Scientific Impact Recognition Award. Sentinel node staging for breast cancer: intraoperative molecular pathology overcomes conventional histologic sampling errors. Am J Surg 194:426-432

62. Mamounas EP (2003) Sentinel lymph node biopsy after neoadjuvant systemic therapy. Surg Clin North Am 83:931-942

63. Xing Y, Foy M, Cox DD et al (2006) Meta-analysis of sentinel lymph node biopsy after preoperative chemotherapy in patients with breast cancer. Br J Surg 93:539-546

64. Clarke M, Collins R, Darby S et al (2005) Effects of radiotherapy and of differences in the extent of surgery for early breast cancer on local recurrence and 15-year survival: an overview of the randomised trials. Lancet 366:2087-2106

65. Patt DA, Goodwin JS, Kuo YF et al (2005) Cardiac morbidity of adjuvant radiotherapy for breast cancer. J Clin Oncol 23:74757482

66. Giordano SH, Kuo YF, Freeman JL et al (2005) Risk of cardiac death after adjuvant radiotherapy for breast cancer. J Natl Cancer Inst 97:419-424

67. Sanders ME, Scroggins T, Ampil FL et al (2007) Accelerated partial breast irradiation in early-stage breast cancer. J Clin Oncol 25:996-1002

68. Fearmonti RM, Vicini FA, Pawlik TM et al (2007) Integrating partial breast irradiation into surgical practice and clinical trials. Surg Clin North Am 87:485-498 (x-xi)
69. Arthur DW, Winter K, Kuske RR et al (2008) A phase II trial of brachytherapy alone after lumpectomy for select breast cancer: tumor control and survival outcomes of Rtog 95-17. Int J Radiat Oncol Biol Phys 72:467-473

70. Vicini FA, Chen P, Wallace $M$ et al (2007) Interim cosmetic results and toxicity using 3D conformal external beam radiotherapy to deliver accelerated partial breast irradiation in patients with early-stage breast cancer treated with breast-conserving therapy. Int J Radiat Oncol Biol Phys 69:1124-1130

71. Benitez PR, Keisch ME, Vicini F et al (2007) Five-year results: the initial clinical trial of MammoSite balloon brachytherapy for partial breast irradiation in early-stage breast cancer. Am J Surg 194:456-462

72. Keisch M, Vicini F (2005) Applying innovations in surgical and radiation oncology to breast conservation therapy. Breast $\mathrm{J}$ 11(suppl 1):S24-S29

73. Donovan E, Bleakley N, Denholm E et al (2007) Randomised trial of standard 2D radiotherapy (RT) versus intensity modulated radiotherapy (IMRT) in patients prescribed breast radiotherapy. Radiother Oncol 82:254-264

74. Pignol JP, Olivotto I, Rakovitch E et al (2008) A multicenter randomized trial of breast intensity-modulated radiation therapy to reduce acute radiation dermatitis. J Clin Oncol 26:2085-2092

75. Harsolia A, Kestin L, Grills I et al (2007) Intensity-modulated radiotherapy results in significant decrease in clinical toxicities compared with conventional wedge-based breast radiotherapy. Int J Radiat Oncol Biol Phys 68:1375-1380

76. Bentzen SM, Agrawal RK, Aird EG et al (2008) The UK Standardisation of Breast Radiotherapy (START) Trial B of radiotherapy hypofractionation for treatment of early breast cancer: a randomised trial. Lancet 371:1098-1107

77. Whelan T, MacKenzie R, Julian J et al (2002) Randomized trial of breast irradiation schedules after lumpectomy for women with lymph node-negative breast cancer. J Natl Cancer Inst 94:11431150

78. Freedman GM, Anderson PR, Goldstein LJ et al (2007) Fourweek course of radiation for breast cancer using hypofractionated intensity modulated radiation therapy with an incorporated boost. Int J Radiat Oncol Biol Phys 68:347-353

79. Formenti SC, Gidea-Addeo D, Goldberg JD et al (2007) Phase III trial of prone accelerated intensity modulated radiation therapy to the breast to optimally spare normal tissue. J Clin Oncol 25:2236-2242

80. Jin JY, Klein EE, Kong FM et al (2005) An improved internal mammary irradiation technique in radiation treatment of locally advanced breast cancers. J Appl Clin Med Phys 6:84-93 